\title{
Beneficial Role of Curcumin in Diseases Treatment: A Mini
}

\section{Review}

\author{
Hassanpour SH${ }^{1 *}$, Dehghani $\mathrm{MA}^{2}$, Alipour SM${ }^{3}$, Karami $\mathrm{SZ}^{4}$ and \\ Dehghani F ${ }^{5}$ \\ 1Young Researchers and Elite Club, Islamic Azad University, Iran \\ ${ }^{2}$ Department of Toxicology, Ahvaz Jundishapour University of Medical Sciences, Iran \\ ${ }^{3}$ Department of Environmental Health, Yasouj University of Medical Sciences, Iran \\ ${ }^{4}$ Department of Biology, Yasouj University, Iran \\ ${ }^{5}$ Department of Genetics, Shiraz University of Medical Sciences, Iran
}

*Corresponding author: Hassanpour SH, Young Researchers and Elite Club, Yasooj Branch, Islamic Azad University, Yasooj, Iran, 7591181963, Tel: +98 9171452844, Fax: +98 7433372379; E-mail: Dr.hossein1366@yahoo.com

\section{Abstract}

Curcumin regulates cellular signaling pathway related to cancer growth, inflammation and apoptosis and due to biological activities can be target DNA, RNA and enzymes. In addition, curcumin has inhibitory effect against MARK, P53, $\beta$-catenin and AKT. It can be consider as a promising agent to treat many diseases but it is important that should be acceptably carried out cellular targeting with curcumin. Here, we reviewed beneficial effects of curcumin in treatment of diseases. Our review study was confirmed that curcumin has potential effect in diseases treatment but there is a dire need for increase of its bioavailability in order to promotion of its efficacy. We suggest that should be design the methods for increase of cellular targeting by curcumin in further studies.

Keywords: Curcumin; Cancer; Inflammation; Apoptosis

\section{Introduction}

Curcumin is considered as a phenolic compound with therapeutic properties including anti-inflammation, antioxidant and anticancer [1]. In previous studies, it has been found that it is safe compound for the body following its administration in patients with cancer and healthy individuals. In fact, its therapeutic effects is more than its side effects [2,3]. However, despite having such a beneficial effect on human biological, it has not very high bioavailability in human due to poor absorption and fast metabolism $[4,5]$. In animal and human, major amount of curcumin is excreted as urine and feces and is absorbed only a small portion of curcumin that combines with glucuronide and sulfate in order to create its conjugated forms in liver. These events lead to reduction of plasma concentrations of free curcumin [2,5-9]. In addition, curcumin is an insoluble compound in water at neutral and basic conditions due to production of phenolate that leads to poor availability in aqueous systems $[10,11]$. Using nanoparticles can increase cellular uptake and stability of curcumin so that solid lipid nanoparticles promote distribution and stability of curcumin in aqueous solutions that lead to improvement of the anticancer effect of 
curcumin [12]. In addition, silica nanoparticle can be a suitable delivery system for curcumin because it has been demonstrated that silica nanoparticle increase anti-tumor properties of curcumin [13]. In this study, we reviewed the beneficial effect of curcumin in order to treatment of diseases.

\section{Review Method}

Here, we searched paper related to role of curcumin in treatment of diseases with keywords such as curcumin and cancer, curcumin and Parkinson's diseases, curcumin and Alzheimer disease, curcumin and histone acetyl transferase (HAT), curcumin and diabetes in databases include web of science, PubMed and Scopus. Then, the papers were fully read and summarized here.

\section{Curcumin Beneficial Effects in Diseases Treatment}

According to a study Yallapu, et al. (2014) PLGA or poly (lactic-co-glycolicacid) combined with curcumin leads to a reduction of tumor cells growth (prostate cancer). Poly (lactic-co-glycolicacid) combined with curcumin had pivotal role in inhibition of beta-catenin expression in tumor cells. In addition, it led to inhibition of antiapoptotic proteins (Mcl-1, Bcl-xL) through suppression of STAT3 and AKT phosphorylation. Down-regulation of miR21 and up-regulation of miR-205 also were showed after treatment with poly (lactic-co-glycolicacid) combined with curcumin. Given that this effects poly (lactic-coglycolicacid) combined with curcumin can be consider as a promising agent to treat cancer due to good delivery into tumor cells [14]. Thiolated chitosan nanoparticle can promote anticancer effect of curcumin because it increases curcumin bioavailability. In addition, treatment of mice with colon cancer by curcumin loaded with thiolated chitosan nanoparticle led to dramatically reduction of cancer cells [15]. Kumar, et al. (2014) examined that treatment of ovarian cancer cells (SKOV-3) with curcuminloaded polymer of 2-hydroxy methyl methacrylate resulted in decrease of cancer cells by arresting of cell cycle in G0/G1 and induction of apoptosis [16]. It has been reported that curcumin-combined silica nanoparticle conjugated with hyaluronic acid results in cytotoxic effects on human colon carcinoma (colo-205) that is due to appropriate delivery of curcumin in to cancer cells through silica nanoparticle conjugated with hyaluronic acid [17]. Curcumin-loaded PLGA-PEG nanoparticle has anti-tumor effects against breast cancer (cell line MCF-7) due to obvious reduction of cancer cells followed by treatment with curcumin-loaded PLGA-PEG nanoparticle [18]. The effect of polysorbate 80-coated cerasomes in order to deliver curcumin in mice with Parkinson's disease was confirmed promotion of cross of blood-brain barrier and subsequently improvement of mice with Parkinson's disease [19]. Curcumin and piperine coated with glycerol monooleate easily pass the blood-brain barrier and prevent formation of alpha synuclein oligomers. In addition, curcumin and piperine coated with glycerol monooleate reduce effect of rotenone as a Parkinson's disease inducer. Curcumin and piperine coated with glycerol monooleate is an important factor in reduction of oxidative stress, apoptosis and autophagy pathway and ultimately protect dopaminergic neurons against damage [20]. A research group was found that aminefunctionalized mesoporous silica nanoparticle could be a good delivery system for curcumin in order to abrogation of Parkinson's disease complications because administration of curcumin-loaded amine-functionalized mesoporous silica nanoparticle leads to reduction of alpha synuclein accumulation [21]. Mathew, et al. (2012) designed a water-soluble nanoparticle (PLGA nanoparticle conjugated with Tet-1 peptide) for transferring of curcumin. Based our study, curcumin loaded-PLGA nanoparticle conjugated with Tet-1 peptide prevents the accumulation of amyloid beta and had antioxidant effect and not cytotoxic effect therefore it can be very effective in the treatment of Alzheimer's disease [22]. It has been showed improvement of memory and reduction of amyloid plaques in mice with Alzheimer's disease after treatment with highly stabilized curcumin nanoparticle according to study conducted by Kin Cheng, et al. (2013). Meanwhile, the presence of high level of curcumin in plasma indicated enhancement of its pharmacokinetic characteristic using the nanoparticle [23]. Curcumin-loaded PLGA nanoparticle is effective in neural stem cell differentiation at rat's hippocampal and sub ventricular. In fact, injection of this compound into hippocampal leads to neurogenesis induction through expression of genes related to cell growth and proliferation (reelin, nestin and Pax6) and differentiation of neurons, such as neurogenin, neuroD1, neuregulin, neuroligin and Stat3. These events ultimately result in improvement of learning and memory due to destruction of beta amyloid [24]. Curcumin-coated magnetic nanoparticle has diagnostic value for Alzheimer's disease because it is a non-invasive diagnostic procedure by MRI and reduces PET disadvantages [25]. Curcuminloaded PLGA nanoparticle is a good agent against Alzheimer's disease due to it protects neurons against oxidative stress by up-regulation of expression of genes like APOE, APOJ, TRX, GLRX REST that indicate its high uptake in neurons [26]. Curcumin inhibits histone acetyl transferase, a main reason of cardiac hypertrophy and heart failure, and prevents hypertrophy $[27,28]$. Furthermore, curcumin protects heart against damage caused by ischemia/reperfusion $[29,30]$. In cells, curcumin 
leads to proteasome-dependent degradation of histone acetyl transferase. This event is main reason for inhibition of histone acetyl transferase by curcumin. In fact, curcumin is a selective inhibitor of histone acetyl transferase [28]. By the mechanism, curcumin prevents excessive increase of ratio heart weight to body weight [31]. Retinopathy occurs in diabetes due to inability of body for elimination of free radicals and pro-inflammatory molecules such as NF- $\mathrm{KB}$, VEGF, and IL1 $\beta$. Curcumin prevents progression of diabetes retinopathy by inhibition of free radicals formation and reduction of inflammation [32]. Curcumin treatment shows great promise as a therapeutic agent. Its beneficial role is being investigated in human clinical trials under different conditions, like multiple myeloma, myelodys plastic syndrome, pancreatic cancer, and colon cancer. Curcumin was also reported to protect against several chemotherapeutic side effects in many studies. Its exposure has been shown to alleviate oxidative stress in PC12 cells exposed to cisplatin [33]. Besides, this unique molecule has been used for a long period as an "herbal general medicine" to relieve discomfort and inflammation associated with different infectious as well as autoimmune diseases [34]. These properties also appear to help this multi-functional molecule to act as a potent anticancer drug. All these beneficial effects of curcumin are summarized in Figure 1.

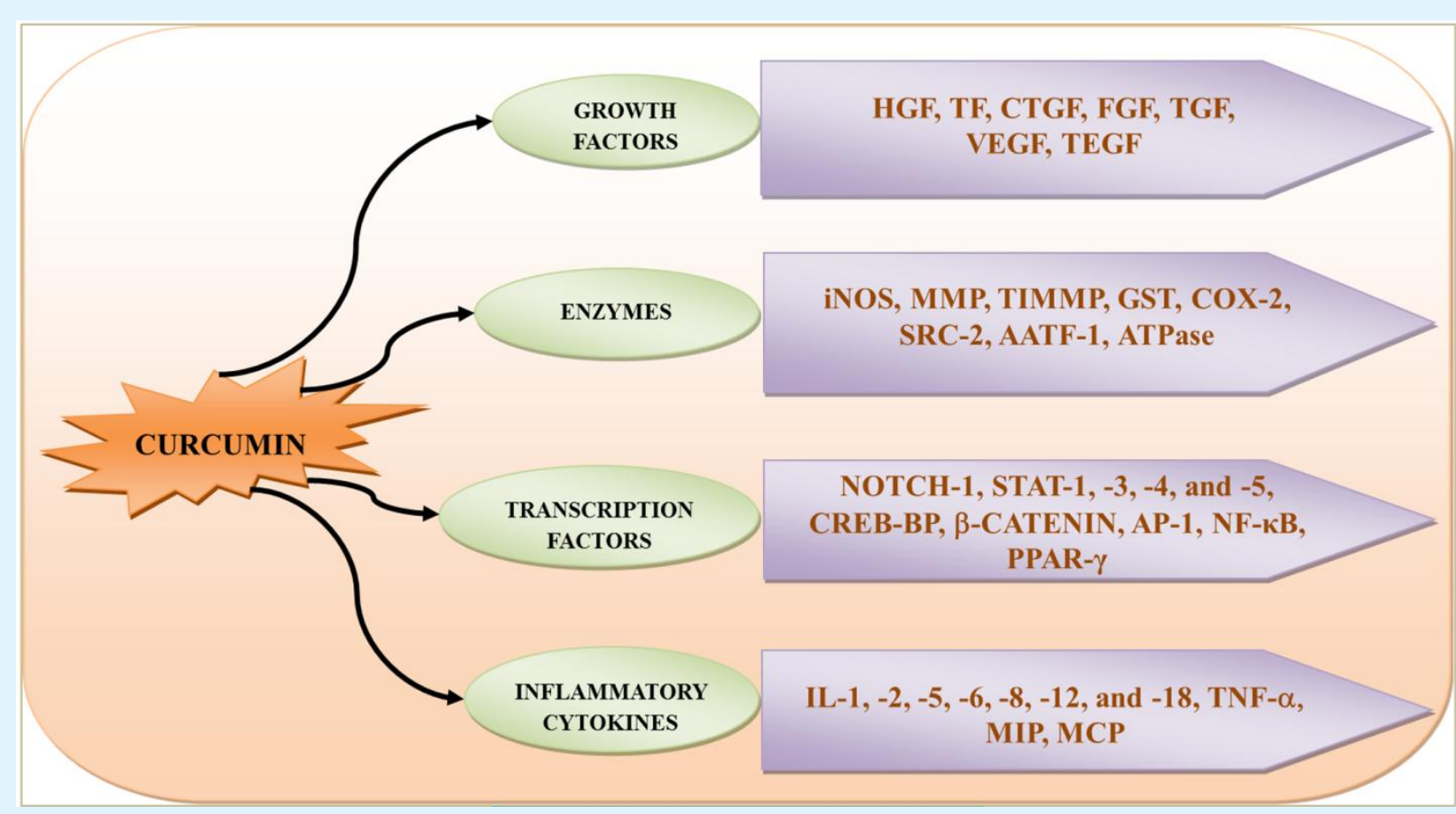

Figure 1: Different signaling molecules, like growth factors, enzymes, transcription factors, and inflammatory cytokines, regulated by curcumin to inhibit the progression of cancer development.

\section{Conclusion}

The use of herbal medicines is increasing in recent years. Thus, efforts to review and revive herbal medicines are reasonable so that to understand their effect mechanism lead to achieve new strategies to treat many diseases. The curcumin is most important component of turmeric. However, its low bioavailability has limited its healing properties. Today, it has been proposed several mechanisms to increase its bioavailability that can be improve its absorption. For example, the nanoparticles can significantly increase curcumin effectiveness. In this review study, we showed that to apply strategies for promotion of curcumin bioavailability lead to increase of its efficacy in treatment of diseases therefore we suggest that design further studies based on this view.

\section{Declarations}

a) Acknowledgements: Us acknowledgements and gratefulness at the beginning and at last is to god who gave us the gift of the mind. The authors thank Young Researchers and Elite Club, Yasooj Branch, Islamic Azad University due to cooperation in this study.

b) Conflict of interest statement: The authors declare that there is no conflict of interest regarding this study. 
c) Financial support and sponsorship: This study was supported by the authors named in this article alone.

d) Contribution of Authors: This work was done by the authors named in this article and all liabilities pertaining to claims relating to the content of this article was borne by the authors named in this article.

e) Ethical approval: This research does not contain any studies with human participants or animals and was performed by the authors alone.

\section{References}

1. Vareed SK, Kakarala M, Ruffin MT, Crowell JA, Normolle DP, et al. (2008) Pharmacokinetics of curcumin conjugate metabolites in healthy human subjects. Cancer Epidemiol Biomarkers Prev 17(6): 1411-1417.

2. Cheng AL, Hsu CH, Lin JK, Hsu MM, Ho YF, et al. (2001) Phase I clinical trial of curcumin, a chemopreventive agent, in patients with high-risk or pre-malignant lesions. Anticancer Res 21(4B): 2895-2900.

3. Lao CD, Ruffin MT, Normolle D, Heath DD, Murray SI, et al. (2006) Dose escalation of a curcuminoid formulation. BMC Complement Altern Med 6(1): 10.

4. Gutierres VO, Campos ML, Arcaro CA, Assis RP, BaldanCimatti HM, et al. (2015) Curcumin pharmacokinetic and pharmacodynamic evidences in streptozotocindiabetic rats support the antidiabetic activity to be via metabolite (s). Evidence-Based Complementary and Alternative Medicine 2015: 678218.

5. Jäger R, Lowery RP, Calvanese AV, Joy JM, Purpura M, et al. (2014) Comparative absorption of curcumin formulations. Nutr J 13(1): 11.

6. Pan MH, Huang TM, Lin JK (1999) Biotransformation of curcumin through reduction and glucuronidation in mice. Drug Metab Dispos 27(4): 486-494.

7. Ireson C, Orr S, Jones DJ, Verschoyle R, Lim CK, et al. (2001) Characterization of metabolites of the chemopreventive agent curcumin in human and rat hepatocytes and in the rat in vivo, and evaluation of their ability to inhibit phorbol ester-induced prostaglandin E2 production. Cancer Res 61(3): 10581064.

8. Sharma RA, Euden SA, Platton SL, Cooke DN, Shafayat A, et al. (2004) Phase I. Clinical Trial of Oral Curcumin:
Biomarkers of Systemic Activity and Compliance. Clin Cancer Res 10(20): 6847-6854.

9. Dulbecco P and Savarino V (2013) Therapeutic potential of curcumin in digestive diseases. World J Gastroenterol 19(48): 9256-7920.

10. Gantait A, Barman T, Mukherjee PK (2011) Validated method for estimation of curcumin in turmeric powder. Indian journal of traditional knowledge 10(2): 247-250.

11. Modasiya M, Patel VM (2012) Studies on solubility of curcumin. International Journal of Pharmacy\& Life Sciences 3(3): 1490-1497.

12. Sun J, Bi C, Chan HM, Sun S, Zhang Q et al. (2013) Curcumin-loaded solid lipid nanoparticles have prolonged in vitro antitumour activity, cellular uptake and improved in vivo bioavailability. Colloids and surfaces B: Biointerfaces 111: 367-375.

13. Gangwar RK, Tomar GB, Dhumale VA, Zinjarde S, Sharma RB, et al. (2013) Curcumin conjugated silica nanoparticles for improving bioavailability and its anticancer applications. J Agric Food Chem 61(40): 9632-9637.

14. Yallapu MM, Khan S, Maher DM, Ebeling MC, Sundram $\mathrm{V}$, et al. (2014) Anti-cancer activity of curcumin loaded nanoparticles in prostate cancer. Biomaterials 35(30): 8635-8648.

15. Anitha A, Deepa N, Chennazhi K, Lakshmanan VK, Jayakumar R (2014) Combinatorial anticancer effects of curcumin and 5-fluorouracil loaded thiolated chitosan nanoparticles towards colon cancer treatment. Biochim Biophys Acta 1840(9): 2730-2743.

16. Kumar SS, Surianarayanan M, Vijayaraghavan R, Mandal AB, Mac Farlane D (2014) Curcumin loaded poly (2-hydroxyethyl methacrylate) nanoparticles from gelled ionic liquid-In vitro cytotoxicity and anticancer activity in SKOV-3 cells. Eur J Pharm Sci 51: 3444.

17. Singh SP, Sharma M, Gupta PK (2015) Cytotoxicity of curcumin silica nanoparticle complexes conjugated with hyaluronic acid on colon cancer cells. Int J Biol Macromol 74: 162-170.

18. Tabatabaei Mirakabad FS, Akbarzadeh A, Milani M, Zarghami N, Taheri Anganeh M, et al. (2016) A Comparison between the cytotoxic effects of pure curcumin and curcumin-loaded PLGA-PEG 
nanoparticles on the MCF-7 human breast cancer cell line. Artif Cells Nanomed Biotechnol 44(1): 423-430.

19. Nisi Z, Huang X, Zhifei D, Zheng H, Fei Y (2016) Localized delivery of curcumin-loaded nanoparticles to the brain for treatment of Parkinson's disease using focused ultrasound. Ultrasonics Symposium (IUS), 2016 IEEE International, 2016: IEEE.

20. Kundu P, Das M, Tripathy K, Sahoo SK (2016) Delivery of Dual Drug Loaded Lipid Based Nanoparticles across the Blood-Brain Barrier Impart Enhanced Neuroprotection in a Rotenone Induced Mouse Model of Parkinson's Disease. ACS Chem Neurosci 7(12): 1658-1670.

21. Taebnia N, Morshedi D, Yaghmaei S, Aliakbari F, Rahimi F, et al. (2016) Curcumin-Loaded AmineFunctionalized Mesoporous Silica Nanoparticles Inhibit $\alpha$-Synuclein Fibrillation and Reduce its Cytotoxicity-Associated Effects. Langmuir 32(50): 13394-13402.

22. Mathew A, Fukuda $T$, Nagaoka $Y$, Hasumura $T$, Morimoto H, et al. (2012) Curcumin loaded-PLGA nanoparticles conjugated with Tet-1 peptide for potential use in Alzheimer's disease. PLoS One 7(3): e32616.

23. Cheng KK, Yeung CF, Ho SW, Chow SF, Chow AH, Baum L (2013) Highly stabilized curcumin nanoparticles tested in an in vitro blood-brain barrier model and in Alzheimer's disease Tg2576 mice. AAPS J 15(2): 324336.

24. Tiwari SK, Agarwal S, Seth B, Yadav A, Nair S, et al. (2013) Curcumin-loaded nanoparticles potently induce adult neurogenesis and reverse cognitive deficits in Alzheimer's disease model via canonical Wnt/ $\beta$-catenin pathway. ACS nano 8(1): 76-103.

25. Cheng KK, Chan PS, Fan S, Kwan SM, Yeung KL, et al. (2015) Curcumin-conjugated magnetic nanoparticles for detecting amyloid plaques in Alzheimer's disease mice using magnetic resonance imaging (MRI). Biomaterials 44: 155-172.
26. Djiokeng Paka G, Doggui S, Zaghmi A, Safar R, Dao L, et al. (2015) Neuronal uptake and neuroprotective properties of curcumin-loaded nanoparticles on SK-NSH cell line: role of poly (lactide-co-glycolide) polymeric matrix composition. Mol Pharm 13(2): 391403.

27. Li HL, Liu C, De Couto G, Ouzounian M, Sun M, et al. Curcumin prevents and reverses murine cardiac hypertrophy. J Clin Invest 118(3): 879-893.

28. Marcu MG, Jung YJ, Lee S, Chung EJ, Lee MJ, et al. (2006) Curcumin is an inhibitor of p300 histone acetylatransferase. Medicinal chemistry 2(2): 169-174.

29. Kapakos G, Youreva V, Srivastava AK (2012) Cardiovascular protection by curcumin: molecular aspects. Indian J Biochem Biophys 49(5): 306-315.

30. Yeh $\mathrm{CH}$, Chen TP, Wu YC, Lin YM, Jing Lin P (2005) Inhibition of $\mathrm{NF \kappa B}$ Activation with Curcumin Attenuates Plasma Inflammatory Cytokines Surge and Cardiomyocytic Apoptosis Following Cardiac Ischemia/Reperfusion. J Surg Res 125(1):109-116.

31. Mito S, Watanabe K, Harima M, Thandavarayan RA, Veeraveedu PT, et al. (2011) Curcumin ameliorates cardiac inflammation in rats with autoimmune myocarditis. Biological and Pharmaceutical Bulletin 34(7): 974-979.

32. Kowluru RA, Kanwar M (2007) Effects of curcumin on retinal oxidative stress and inflammation in diabetes. Nutr Metab 4(1): 8.

33. Mendonca LM, da Silva Machado C, Teixeira CC, de Freitas LA, Bianchi Mde L, et al. (2013) Curcumin reduces cisplatin-induced neurotoxicity in NGFdifferentiated PC12 cells. Neurotoxicology 34: 205211.

34. Ghosh A, Sil PC (2007) Anti-oxidative effect of a protein from Cajanus indicus $\mathrm{L}$ against acetaminophen-induced hepato-nephro toxicity. J Biochem Mol Biol 40(6): 1039-1049. 